Review article

\title{
Avian immunosenescence
}

\author{
Emma T. Lavoie* \\ Department of Animal and Avian Sciences, University of Maryland, Building 142, College Park, MD \\ 20742, USA; *Author for correspondence (e-mail: elavoie@umd.edu; fax: +301-314-9059)
}

Received 2 August 2005; accepted in revised form 30 August 2005

Key words: avian aging, immunosenescence, Japanese quail, Coturnix japonica

\begin{abstract}
Immunosenescence, the aging of the immune system, is well studied in humans. Mammalian immune systems become less capable of fighting pathogens and individuals become more susceptible to infection and cancer in their elder years. Little is currently published on avian immunosenescence even though avian immune function has been well characterized and birds have been critical models in the study of immunology. The value of birds in the study of aging has been well established. Evidence demonstrates a decline in cell-mediated and (or) humoral immune function with age in four species: the barn swallow, collared flycatcher, ruff and Japanese quail. These studies suggest that birds may experience age-related changes in immune function similar to humans. Therefore, avian aging models should be evaluated for changes in immune function and comparisons made between short- and long-lived birds. Specifically, data on age-related changes in innate immunity are lacking for birds and should be characterized. These data will strengthen the use of avian models for the study of aging.
\end{abstract}

\section{Introduction}

Basic research on immunosenescence, or the aging of the immune system, in non-mammalian classes of vertebrates has obvious value; birds especially fit a valuable niche in biomedical research and have produced much valuable data on separate studies of immunity and aging (Glick 1977; Holmes and Ottinger 2003). However, comparatively few avian immunosenescence studies have been published relative to mammals. Aging of the immune system and changes in inflammatory response have become of great interest in the literature in recent years in reference to disease and quality of life. Avian models can potentially be useful in investigations of life span. Furthermore, disease outbreaks such as West Nile virus and avian influenza encourage further avian immune research.

\section{Avian immunology}

The avian immune system is generally very similar to the mammalian immune system and can be split into three branches: innate, cell-mediated and humoral immunity. The innate immune system includes natural killer cells and phagocytic leukocytes. These cells elicit non-specific defenses against pathogens, by recognizing and destroying infected cells before specific immune responses develop to combat the infection. Cell-mediated immunity (CMI) refers to the actions of T-lymphocytes, or those lymphocytes originating in the thymus. Simply defined, T-cells may be cytotoxic (CD8 T-cells) or helper (CD4 T-cells) in action. CD8 T-lymphocytes kill virally infected cells. CD4 T-lymphocytes differentiate into either T-helper type 1 or T-helper type 2 lymphocytes. The former play a role in macrophage activation 
and CD8 T-lymphocyte stimulation, the latter stimulate antibody isotype switching in B-lymphocytes. Assays of T-cell function are usually made by measuring lymphocyte proliferation to polyclonal $\mathrm{T}$ cell mitogens, delayed type hypersensitivity or lymphocyte cytotoxicity. Humoral immunity refers to the production of antibodies by B-lymphocytes that originate and mature in bone marrow in mammals but which mature in the bursa of Fabricius in birds. The presence of the bursa of Fabricius in birds is the biggest difference in immune system structure between birds and mammals. Measurements of humoral immunity are usually made by testing antibody production to a known antigen, either T-helper-celldependent, such as sheep red blood cells (SRBC), or T-helper-cell-independent, such as trinitrophenol-lipopolysaccharide (TNP-LPS). In mammals, antibodies are separated into five immunoglobulin (Ig) subclasses: $\operatorname{IgM}, \operatorname{IgD}, \operatorname{IgG}, \operatorname{Ig} \mathrm{A}$, and $\operatorname{IgE}$. CMI and humoral immune responses are antigen-specific. That is, only those lymphocytes that are specific to an antigen will initiate an immune response through proliferation and differentiation. An increase in immune function may result in allergy or autoimmunity, while a decrease in immune function results in impaired ability to fight disease (Janeway and Travers 1997).

Avian lymphocyte precursors originate in the bone marrow and other compartments such as yolk in the embryo, then migrate to the thymus or bursa of Fabricius ('bursa') to mature into T-cells or B-cells respectively. With the exception of the maturation of B-cells in the bursa, avian T- and B-cells develop and function in a similar manner to those in mammals (Sharma 1991; Chen et al. 1996). Thymocytes mature in the thymus while undergoing proliferation and maturation. Those few $(<5 \%)$ that survive the maturation processes exit to the periphery as $\mathrm{CD}^{+}$or $\mathrm{CD}^{+}$('single positive') T-cells (Janeway and Travers 1997). Avian B-lymphocytes mature in the bursa, an organ unique to birds, while mammalian B-lymphocytes mature in bone marrow. In chickens, B-cell precursors enter the bursa (a gut-associated organ), between embryonic days 8 and 14, proliferate and develop surface immunoglobulin through gene conversion (Funk and Thompson 1996; Reynaud and Weill 1996). B-cells exit the bursa to the periphery only a few days before hatch and rates of emigration increase until the third week post-hatch. Post-hatch, antigens from digested foods may enter the bursa due to reverse peristalsis of the hindgut. These antigens may drive normal post-hatch B-cell development (Glick 2000; Sayegh et al. 1999, 2000). IgM, IgG, IgA and IgY antibodies have been described in birds, but identical roles to mammalian forms have not been confirmed. In avian studies, common measures of in vivo immune function are the phytohemagglutinin (PHA) skin test response and antibody response to foreign red blood cells (Fairbrother et al. 2004). The PHA skin test is a T-cell-dependent inflammatory response.

\section{Immunosenescence in humans}

Current literature on humans describes a decline or functional change in immunity with aging, driven initially by the atrophy of the thymus gland. A reduction in ability of T-cells to proliferate, reduced B-cell responses and altered activity of immune associated cells and cytokines (Malaguarnera et al. 2001) are also documented. Coinciding with the decline in immunity, older humans experience an increase in rates of cancer and autoimmunity over those of younger individuals. Furthermore, the elderly have poor responses to influenza vaccination (Potter et al. 1999, reviewed in Plackett et al. 2004). Specific changes in immune function documented in the elderly include an increase in the ratio of memory to naïve lymphocytes in the blood $\left(\mathrm{CD} 45 \mathrm{RO}^{+}\right.$:

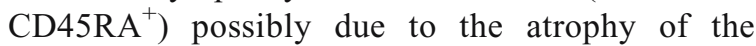
thymus, an alteration in T-helper lymphocyte activity, especially in a decline of interleukin production, and a decrease in the ratio of T-helper to T-suppressortype cells (Malaguarnera et al. 2001). In the innate branch of the immune system, natural killer cell activity (but not number) and tumoricidal ability decrease with age, as does the microbiocidal function of macrophages and polymorphonuclear cells. This decline in turn leads to longer-lasting infections in the elderly (Plackett et al. 2004; Malaguarnera et al. 2001). Dendritic cells, important in antigen presentation early in an immune response, are less stimulatory of lymphocytes, leading to changes in humoral immunity (Plackett et al. 2004). Immunoglobulin production decreases, although B-cell number does not change; the Ig production changes are likely related to failing T-cell function (Malaguarnera et al. 2001). 


\section{Immunosenescence in birds}

Measurements of age-related changes in immunity have been made in at least four bird species: barn swallows (Hirundo rustica), collared flycatchers (Ficedula albicollis), common terns (Sterna hirundo) and ruffs (Philomachus pugnax) (Saino et al. 2002; Apanius and Nesbit 2003; Cichón et al. 2003; Lozano and Lank 2003; Saino et al. 2003). The studies on barn swallows, flycatchers and ruffs were made in relation to ecological questions. No comprehensive studies on avian immunosenescence have been published; that is to say, no study has incorporated a full-scale evaluation of innate, cellmediated and humoral immunity in relation to aging in birds. Although some work has been done in early life stages, even the chicken has not been well evaluated for changes in immunity over its entire life span. McCorkle and Glick (1979) evaluated immune function in groups of chickens aged 1, 3, 6, 12, 18 and 24 months. Anti-red-blood-cell antibody responses were highest in birds at one month of age and were lower at all other ages tested in males, and marginally lower in females compared to values at 1 month. T-independent antigen (Brucella abortus) responses were lowest at 1 month and consistently higher in the same range at all other ages tested, with no differences between males and females. However, this study did not encompass the potential life span of chickens and rather evaluated a single immune function in juvenile and young reproductive birds.

There is evidence of decreased humoral immunity in aging passerines. Barn swallows were immunized with Newcastle disease virus (NDV) vaccine twice in successive breeding seasons. Females of one to 3 years old showed an age-related decline in anti-NDV response during the first year, and both females ( 2 to 4 years old) and males ( 2 to 6 years old) showed an age-related decline in secondary anti-NDV response in the second year (Saino et al. 2003). Female collared flycatchers of three age groups (young: 1 year, middle-aged: 3 years and old: 5-6 years) were immunized with sheep red blood cells (SRBC) while feeding their nestlings. These birds demonstrated an age-related decline in anti-SRBC response with an approximate two-fold decline in antibody titer between each age group from youngest to oldest (Cichón et al. 2003). The authors point out that even though older individuals had lower immune responses, this is possibly related only to novel antigens. The survival of the older birds to 'old age' is probably related to good memory responses to antigens encountered at a younger age and(or) compensation of declining humoral immune function by other branches of the immune system which were not analyzed in this study. Furthermore, Cichón et al. (2003) argue that their data disproves the idea that declines in immune function measured in domestic or laboratory-reared species are a result of artificial laboratory conditions. One of the remaining questions in the studies by Saino and Cichün is whether breeding was contributing to differences in immune function in age classes. That is to say, were older birds putting more resources into breeding than younger birds and thereby compromising resources for immune function? Apanius and Nesbit (2003) show evidence in a longer lived, fish-eating bird, the common tern, that serum IgG levels do not change with age. They note that, based on the mammalian literature, an increase $\operatorname{IgG}$ with age would be expected.

Evidence is also available that cell-mediated immunity (measured as phytohemagglutinin skin response) decreases with age in birds. In barn swallows, older females produced chicks that had decreased Tcell-mediated immunity compared to chicks of younger females (Saino et al. 2002). In a study of a captive breeding flock of ruffs, T-cell-mediated immunity declined with age regardless of gender. Interestingly, in ruffs, male immunity decreased during the breeding season and was more variable than female immunity (Lozano and Lank 2003).

The Japanese quail (Coturnix coturnix japonica) has a similar aging pattern to that of laboratory rodents and has been studied as a short-lived aging model. The quail was named as a model species with special potential for aging studies by Holmes and Ottinger (2003) because of its domestication, ease and excellence of breeding, short life span with rapid aging (especially reproductive aging), and extensive literature available on its behavior and neuroendocrinology (Balthazart and Adkins-Regan 2002). Japanese quail are reproductively mature at two months of age and show signs of reproductive aging by 18 months. Clinical symptoms of aging include a decline in ovarian function and egg laying, decline in male sexual behavior, tumors of the reproductive organs, brittle bones and cataracts (Holmes and Ottinger 2003). However, little to nothing is known about the aging of quail immune systems. 
Table 1. Differences in immune responses of different aged Japanese quail: mean (standard error) $N$.

\begin{tabular}{|c|c|c|c|c|}
\hline & \multicolumn{4}{|c|}{ Age (months) } \\
\hline & 0.5 & 1.5 & 10 & 28 \\
\hline & Juvenile & Pubescent & Reproductive adult & Aging \\
\hline PHA skin response (mm) & NM & $0.62(0.02) 41$ & $0.75(0.05) 30$ & $0.47(0.03) 32$ \\
\hline Anti-RBC titer $\left(\log _{2}\right)$ & NM & $2.8(0.6) 41$ & $5.2(0.5) 28$ & $4.5(0.6) 29$ \\
\hline Increase in anti-flu titer $\left(\log _{2}\right)$ & $1.4(0.2) 10$ & $0.22(0.4) 9$ & $2.9(0.3) 9$ & $1.2(0.3) 9$ \\
\hline
\end{tabular}

Lavoie et al. (unpublished data) characterized the immune function of different age groups of Japanese quail with the hypothesis that immune function declines with age, and, time to recover from an infection is longer in older birds compared to younger birds. Groups of quail were evaluated for in vivo immune function and challenged with influenza (H9N2 A/Quail/Hong Kong/A28945/88) at three age classes: pubescent (6 weeks old), reproductive adult (10 months old) and aging (28 months old). A fourth group was added (juvenile, 2.5 weeks old) for the influenza challenge. Reproductive adult birds gave the highest PHA skin response, followed by the pubescent group, with the aging group 38\% lower than reproductive adults. Reproductive birds had the highest anti-red blood cell titers, pubescent birds' mean response was four fold lower than those of the reproductive adults and the aging birds were intermediate in response between adults and pubescent birds. The difference in antiviral antibody response between primary and secondary infections was strongly affected by age; when expressed as the difference between primary and secondary responses, adults had greater increase in total antibody than all other groups (Table 1). This experiment demonstrated a clear age-related difference in immune function in Japanese quail that has similarities to age-related immunity in humans, in that younger and older animals had weaker immune responses compared to young adults.

\section{Future work needed}

In-depth analyses of immunosenescence, in a variety of domestic or wild birds, are needed. An interesting comparison would be the immune function changes with age between short and long-lived birds because birds have differing body temperatures to mammals and exhibit varying basal metabolic rates, oxidative damage and patterns of physiological aging. Future in-depth investigations should include more specific cellular function such as proliferation, cytotoxicity, inflammatory response, cytokine expression, type I versus type II immunity, cell type analysis, and evaluation of innate immunity. Furthermore, the histology of the primary immune organs in birds, the thymus and bursa, should be compared and contrasted between species in relation to immunosenescence. The age-related atrophy of these organs may differ temporally in different species and possibly drive changes in immune function across the life span.

\section{References}

Apanius V and Nesbit ICT (2003) Serum immunoglobulin G levels in very old common terns Sterna hirundo. Exp Gerontol 38(7): 761-764

Balthazart J and Adkins-Regan E (2002) Sexual differentiation of brain and behavior in birds. In: Pfaff D (ed) Hormones, Brain and Behavior, Vol. 4, p 881. Academic, San Diego CA

Chen CH and Six A et al. (1996) T Cell Receptors and T Cell Development. Springer, Berlin Heidelberg New York

Cichón M and Sendecka J et al. (2003) Age-related decline in humoral immune function in collared flycatchers. J Evol Biol 16(6): 1205-1210

Fairbrother A, Smits J and Grasman KA (2004) Avian immunotoxicology. J Toxicol Environ Health, B 7: 105-137

Funk PE and Thompson CB (1996) Current concepts in chicken B cell development. Immunology and developmental biology of the chicken. Springer, Berlin Heidelberg New York

Glick B (1977) The bursa of Fabricius and immunoglobulin synthesis. Int Rev Cytol 48: 345-402

Glick B (2000) Immunophysiology. Sturkie's Avian Physiology. G. C. Whittow. Academic, San Diego

Holmes DJ and Ottinger MA (2003) Birds as long-lived animal models for the study of aging. Exp Gerontol 38: 1365-1375 
Janeway CA and Travers P (1997) Immunobiology: the immune system in health and disease. Taylor and Francis, London

Lozano GA and Lank DB (2003) Seasonal trade-offs in cellmediated immunosenescence in ruffs (Philomachus pugnax). Proc R Soc Lond, B 270: 1203-1208

Malaguarnera L, Ferlito L, Imbesi RM, Gulizia GS, Ki Mauro S and Maugeri D et al. (2001) Immunosenescence: a review. Arch Gerontol Geriatr 32: 1-14

McCorkle F and Glick B (1979) The effect of aging on immune competence in the chicken:antibody mediated immunity. Poultry Sci 59: 669-672

Plackett T and Boehmer E et al. (2004) Aging and innate immune cells. J Leukoc Biol 76: 291-299

Potter JM, O'Donnel B, Carman WF, Roberts MA and Stoll DJ (1999) Serological response to influenza vaccination and nutritional and functional status of patients of geriatric medical long term care. Age aging 28: 141-145
Reynaud C-A and Weill JC (1996) Postrearrangement Diversification Processes in Gut-Associated Lymphoid Tissues. Immunology and Developmental Biology of the Chicken. Springer, Berlin Heidelberg New York

Saino N, Ambrosini R, Martinelli R and Moller AP (2002) Mate fidelity, senescence in breeding performance and reproductive trade-offs in the barn swallow. J Anim Ecol 71: 309-319

Saino N and Ferrari RP et al. (2003) Humoral immune response in relation to senescence, sex and sexual ornamentation in the barn swallow (Hirundo rustica). J Evol Biol 16(6): 1127-1134

Sayegh CE and Rao MA et al. (1999) Avian B cell development: lessons from transgenic models. Vet Immunol Immunopathol 72: 31

Sayegh CE and Demaries SL et al. (2000) The chicken B-cell receptor complex and its role in avian B-cell development. Immunol Rev 175: 187-200

Sharma JM (1991) Overview of the avian immune system. Vet Immunol Immunopathol 30: 13 